\title{
eJRIEPS
}

Ejournal de la recherche sur l'intervention en éducation physique et sport

$30 \mid 2013$

Varia

\section{Les ressources exploitées par les élèves pour agir dans un format pédagogique en atelier : Une étude empirique lors de leçons de musculation en Education Physique et Sportive}

Student Resources for Acting in the Educational Workshop Format: An Empirical Study During Resistance-Training Lessons in Physical Education

David Adé, Mathieu Picard et Jacques Saury

\section{OpenEdition}

Journals

Édition électronique

URL : http://journals.openedition.org/ejrieps/2374

DOI : $10.4000 /$ ejrieps. 2374

ISSN : 2105-0821

Éditeur

ELLIADD

\section{Référence électronique}

David Adé, Mathieu Picard et Jacques Saury, « Les ressources exploitées par les élèves pour agir dans un format pédagogique en atelier : Une étude empirique lors de leçons de musculation en Education Physique et Sportive », eJRIEPS [En ligne], 30 | 2013, mis en ligne le 01 juillet 2013, consulté le 10 décembre 2020. URL : http://journals.openedition.org/ejrieps/2374 ; DOI : https://doi.org/10.4000/ ejrieps.2374

La revue eJRIEPS est mise à disposition selon les termes de la Creative Commons Attribution 4.0 International License. 


\section{Les ressources exploitées par les élèves pour agir dans un format} pédagogique en atelier: Une étude empirique lors de leçons de musculation en Education Physique et Sportive

David Adé ${ }^{*}$ Mathieu Picard ${ }^{\star *} \&$ Jacques Saury***

* Laboratoire du Centre d'Étude des Transformations des Activités Physiques et Sportives EA3832, Université de Rouen, France.

** Laboratoire du Centre d'Étude des Transformations des Activités Physiques et Sportives EA3832, Université de Rouen, France.

*** Laboratoire «Motricité, Interactions, Performance " EA4334, Université Nantes, France.

Résumé

Cette étude s'intéresse aux ressources situationnelles que les élèves utilisent lors de leçons d'éducation physique en musculation conçues dans un format pédagogique en ateliers, en explorant les liens entre l'arrangement spatial des ateliers, la nature des objets matériels présents dans chaque atelier et les interactions entre les élèves. Elle a été conduite selon le cadre théorique et méthodologique du " cours d'action ». Deux types de données ont été recueillis : (a) des données d'enregistrement audio et vidéo des élèves en classe et (b) des données de verbalisations lors d'entretiens d'autoconfrontation. Les résultats révèlent chez les élèves réalisant les exercices (a) l'existence de quatre préoccupations typiques dirigées vers des ateliers distants associées à trois actions typiques dans l'activité des élèves, et (b) des relations entre ces actions typiques et le type d'appareil de musculation présent dans l'atelier. Ces résultats accréditent l'idée de la classe comme un système éco-social.

Mots clés : cours d'expérience, éducation physique et sportive, ressources distribuées, objets matériels.

\section{Introduction}

Les façons d'agencer le travail des élèves dans la classe contribuent à l'efficacité des enseignants, à la participation et responsabilisation des élèves dans les tâches scolaires, 


\section{eJRIEPS 30 juillet 2013}

et à la configuration des relations enseignants-élèves (pour une synthèse, voir Evertson \& Weinstein, 2006). Une des particularités de l'enseignement de l'Education Physique et Sportive (EPS) réside cependant dans le fait que les enseignants doivent le plus souvent agencer matériellement leur espace de travail de façon ad hoc pour organiser l'activité des élèves dans la classe et conduire leur enseignement. En effet, cet espace de travail n'est pas toujours prédéfini dans l'espace du gymnase ou du stade, comme il l'est dans les salles de classes ordinaires. Les enseignants génèrent ainsi des «formats pédagogiques » (Gal-Petitfaux \& Durand, 2001) dont les plus caractéristiques en EPS sont les colonnes, les files indiennes, les vagues ou encore les ateliers de travail. Le format en ateliers se caractérise par le groupement d'un nombre réduit d'élèves (entre deux et six en général), auxquels l'enseignant attribue une tâche spécifique à réaliser dans un espace précisément délimité, en utilisant (ou non) des objets matériels particuliers pour réaliser la tâche. Ce format est particulièrement répandu en EPS, car il présente divers avantages du point de vue des enseignants. II leur permet notamment d'optimiser l'utilisation du matériel pédagogique et des équipements sportifs et de structurer l'espace et le temps de la leçon d'une façon qui favorise la supervision et le guidage des apprentissages des élèves (Cizeron \& Gal-Petitfaux, 2006). II offre en particulier aux enseignants davantage d'opportunités de différencier leurs interventions auprès des élèves que les autres formats pédagogiques dans des classes avec une forte hétérogénéité (Lotan, 2006). II facilite aussi la mise en œuvre de différentes formes de travail coopératif entre élèves (e.g., Dyson, Griffin \& Hastie, 2004; Dyson \& Grineski, 2001; Ensergueix \& Lafont, 2010, 2011; Ward \& Lee, 2005). En déléguant aux élèves une partie de la responsabilité de l'organisation du travail et de la supervision des apprentissages dans chaque groupe, ce format pédagogique permet à l'enseignant de se délester ponctuellement du management d'une partie de la classe. Lorsqu'il intervient auprès d'un élève ou d'un groupe d'élèves, les autres élèves continuent à réaliser leur tâche de façon autonome dans les différents ateliers. La récurrence du format pédagogique en ateliers dans les pratiques des enseignants d'EPS révèle l'importance qu'ils accordent à la structuration des espaces de travail des élèves et à l'organisation du collectif d'élèves de la classe à des fins d'efficacité pédagogique. Le système des interactions entre l'enseignant et les élèves ou entre les élèves est par conséquent fortement lié au système d'organisation des tâches d'apprentissage, comme l'ont montré les recherches conduites dans le paradigme de l'écologie de la classe (e.g., Hastie \& Siedentop, 1999, 2006). Dans cette perspective, l'organisation spatiale des ateliers dans la classe influence l'écologie de la classe, et plus 


\section{eJRIEPS 30 juillet 2013}

particulièrement, les transactions entre l'enseignant et les élèves, mais aussi entre les élèves. S'inscrivant dans le prolongement du paradigme de l'écologie de la classe, d'autres recherches conduites en référence à une perspective située de l'EPS se sont également attachées à analyser la dynamique et l'inscription contextuelle et sociale de l'activité de l'enseignant et des élèves (Durand, 1998; Kirk \& Macdonald, 1998; Rovegno, 2006). Certaines de ces études se sont intéressées à la façon dont les enseignants agençaient dans l'espace les différents ateliers. Elles ont pointé les relations entre l'agencement spatial des ateliers, et la nature des consignes et des feedback des enseignants, ou leur fréquence (Durand, 1998, 2001). Une nette distinction a été établie, par exemple en gymnastique, entre des ateliers disposés en ligne, lorsqu'ils étaient placés les uns derrière les autres, ou lorsqu'ils étaient symétriquement opposés. Dans le premier cas, la disposition spatiale des ateliers s'accompagnait d'une tendance de l'enseignant à réitérer les feedback adressés à un élève dont les réalisations traduisaient un comportement remarquable aux élèves qui suivaient immédiatement cet élève, selon un effet de "contamination " des interventions de l'enseignant sur les suivantes, de proche en proche, jusqu'à être à nouveau surpris par une réponse atypique d'élève. Dans le deuxième cas, la disposition spatiale des ateliers s'accompagnait fréquemment d'une " dérive " des interventions des enseignants, s'éloignant progressivement du contenu des instructions initiales. Ces recherches ont clairement révélé la place de l'organisation spatiale du travail des élèves et de l'enseignant dans la dynamique de leur activité. Cependant elles ont considéré le format pédagogique en atelier d'une façon générique, indépendamment des particularités concrètes de chaque atelier. Elles ont négligé la façon dont les objets présents dans chaque atelier, ainsi que leur disposition spatiale dans le gymnase, étaient susceptibles d'orienter l'activité individuelle et collective des élèves, et les interventions de l'enseignant. S'inscrivant dans le programme scientifique et technologique du cours d'action (Theureau, 2004) ces travaux conçoivent l'activité comme indissolublement individuelle et collective. En d'autres termes l'activité individuelle de chaque élève est toujours en relation constitutive avec autrui (les autres élèves et l'enseignant) et l'activité collective en classe se construit à partir des activités individuelles (Theureau, 2006). Poursuivant dans cette veine, des travaux récents ont décrit la place et le rôle des objets matériels dans l'activité des élèves en EPS (Adé, Veyrunes \& Poizat, 2009; Adé, Jourand \& Sève, 2010). Leurs résultats ont montré que les objets contribuaient à perturber, stabiliser ou ritualiser l'activité collective en classe. Ils constitueraient des ressources ouvrant à chaque instant des possibles pour la construction de l'activité 


\section{eJRIEPS 30 juillet 2013}

collective en classe. Ces résultats convergent avec ceux ayant déjà pointé le caractère situé des apprentissages et l'importance des artefacts dans des situations d'apprentissages (Bril 2002; Lave \& Wenger, 1991; Norman, 1993; Winn, 2002). Ils accréditent l'hypothèse d'une inscription contextuelle de l'activité des acteurs en classe (Gal-Petitfaux, Sève, Cizeron \& Adé 2010; Jonassen \& Land 2000 ; Saury, Adé, GalPetitfaux, Huet, Sève \& Trohel, 2013), indissociable de l'arrangement spatial de la leçon et des objets matériels qui composent les ateliers de travail.

La présente étude vise à approfondir la question de l'inscription contextuelle des activités de l'enseignant et des élèves au cours des leçons d'EPS. Dans un format pédagogique en ateliers, les enseignants prescrivent le plus souvent un travail différent à réaliser sur chaque atelier, et mettent à disposition des élèves dans chaque atelier les objets les plus appropriés pour faciliter leurs apprentissages. Ces ateliers disposés à différents endroits dans l'espace de la classe constituent des unités de travail distinctes, relativement isolées les unes des autres. Cet espace demeure cependant un espace commun pour les élèves de la classe. Le contexte des activités et apprentissages scolaires est inévitablement collectif et public (Doyle, 1986) : chaque élève agit sous le regard des autres élèves et de l'enseignant, et peut réciproquement à tout moment observer les comportements des autres dans la classe. Cette visibilité mutuelle renforce le caractère indissociable des dimensions individuelles et collectives des situations d'apprentissages (Salomon \& Perkins, 1998). Plusieurs études ont récemment montré que cette visibilité mutuelle favorisait l'émergence d'interactions spontanées d'apprentissage entre les élèves, et la prise en compte de l'activité des autres élèves et de l'enseignant comme des ressources distribuées pour apprendre (De Keukelaere, Guérin, \& Saury, 2008; Guillou \& Durny, 2008; Huet \& Saury, 2011). Elles ont aussi montré que le caractère manifeste de l'activité des autres élèves et de l'enseignant en classe permettait aux élèves de s'ajuster de façon subtile aux attentes de l'enseignant concernant l'engagement dans le travail scolaire (GalPetitfaux \& Vors, 2010), ou de faire face collectivement aux situations d'évaluation scolaire (Saury, Huet, Rossard \& Sève, 2010; Saury \& Rossard, 2009).

C'est pourquoi, en nous appuyant sur ces résultats, il nous a semblé intéressant d'étudier la façon dont les élèves d'une classe sont (ou non) susceptibles d'interagir entre eux entre des ateliers distincts. Autrement dit, il s'agit de nous intéresser aux ressources situationnelles que les élèves utilisent dans un format pédagogique en ateliers, en explorant les liens entre les interactions entre les élèves, l'arrangement spatial des ateliers, et la nature des objets matériels présents dans chaque atelier. Pour cela nous 


\section{eJRIEPS 30 juillet 2013}

avons choisi d'étudier l'enseignement de la musculation en Lycée, dans la mesure où cette discipline sportive se pratique classiquement sous la forme d'ateliers, et exploite des appareillages variés (e.g., squat, développé couché, etc.). Lors de cet enseignement, une rotation des élèves sur les différents ateliers (respectivement associés à des appareils de musculation spécifiques) est organisée, afin que les différents groupes d'élèves puissent pratiquer tour à tour sur chacun des ateliers. L'arrangement spatial des différents appareils de musculation structure donc la classe en petits groupes d'élèves plus ou moins distants les uns des autres dans un espace commun.

\section{Cadre théorique}

Cette étude, inscrite dans le domaine de l'ergonomie cognitive des situations d'intervention (Saury et al., 2013) en référence au programme scientifique du cours d'action (Theureau, 2004, 2006), s'attache à décrire la dynamique de construction des significations par des acteurs dans le cours de leur activité à des fins d'aide à la conception d'environnement d'apprentissage. Elle est en accord avec les présupposés du paradigme de "l'action ou de la cognition située " (Hutchins, 1995; Kirshner \& Whitson, 1997; Lave, 1988; Robbins \& Aydede, 2008; Suchman, 1987) qui en prenant en compte l'environnement dans l'analyse de l'activité, ont conduit à mettre en avant la complexité et les propriétés dynamiques des situations d'intervention et le caractère émergent de l'activité (Gal-Petitfaux et al., 2010 ; Saury et al., 2013). Ce choix paradigmatique nous semble pertinent pour l'analyse et la compréhension des situations d'enseignement en EPS, notamment lorsqu'il s'agit de questionner les liens entre l'activité d'élèves, l'arrangement spatial d'un format pédagogique de travail et des objets matériels.

Le programme scientifique du cours d'action repose entre autres sur trois présupposés relatifs à l'activité humaine. Le premier présupposé est que l'activité est autonome, c'est-àdire qu'elle consiste en des interactions asymétriques entre un acteur et son environnement caractérisant une dynamique de couplage structurel (Maturana \& Varela, 1987). Dans le cadre de notre étude cela signifie que tous les élèves n'interagissent pas de la même façon avec les composants humains et matériels de l'environnement dans lequel ils évoluent, chacun interagissant avec ceux qui sont significatifs pour lui, et qui lui permettent de déployer de façon autonome une action unique et non totalement prédéterminée. Le deuxième présupposé est que l'activité est située dynamiquement, c'est-à-dire qu'elle est indissociable de la situation dans laquelle elle prend forme et l'acteur participe à la construction de cette situation. Le couplage structurel 


\section{eJRIEPS 30 juillet 2013}

acteur/environnement se transforme en permanence au cours de l'activité des acteurs. Dans le cadre de notre étude cela signifie que l'activité des élèves qui émerge de ce couplage structurel, découle d'un effort d'adaptation au contexte dont les éléments significatifs pour les élèves constituent des ressources pour agir. Ces éléments significatifs (par exemple, la proximité ou l'éloignement de l'enseignant; les caractéristiques physiques d'un appareillage ; des bruits) peuvent être localisés, dans le cadre d'un format en atelier de travail, dans les limites spatiales de l'atelier, ou au-delà de ces limites (potentiellement dans tout l'espace de la classe, incluant les autres ateliers). En ce sens l'activité doit être étudiée in situ (Lave, 1988). Le troisième présupposé est que l'activité est vécue, c'est-à-dire qu'elle s'accompagne d'une expérience " en première personne " (Varela \& Shear, 1999), dont l'acteur peut rendre compte, au moins en partie, moyennant des conditions méthodologiques et de collaboration favorables, et qui constitue son "cours d'expérience" (Theureau, 2006). Par hypothèse, ce cours d'expérience est constitutif de toute activité. Le cours d'expérience est «la construction du sens pour l'acteur de son activité au fur et à mesure de celle-ci » (Theureau, 2006, p.48). Cette définition est fondée sur le postulat que ce niveau de l'activité (l'histoire de son activité montrable, racontable et commentable par l'acteur) permet de parvenir à une « description symbolique acceptable » (Varela, 1989, p.184) de la dynamique du couplage structurel d'un acteur avec sa situation. Traduisant la nature sémiotique de la cognition humaine (Whitson, 1997), le cours d'expérience est constitué d'un enchaînement d'unités significatives émergeant de l'interaction de l'acteur avec son environnement (Theureau, 2006), dont chaque acteur est susceptible de rendre compte lorsqu'il est invité à raconter l'histoire de son activité passée. Ces unités peuvent être des actions (pratiques ou de communication), des sentiments, des interprétations ou des focalisations. La restitution de cet enchaînement permet de reconstruire la dynamique de la construction de l'activité de l'acteur en relation avec la situation dans laquelle il est engagé.

Dans le cadre de notre étude, l'analyse de chaque cours d'expérience vise à rendre compte du flux de significations qui émerge des actions de l'élève et accompagne le déroulement de son activité. Renseigner ces significations c'est, entre autres, accéder aux ressources mobilisées par les élèves lorsqu'ils interagissent entre eux dans un format pédagogique en atelier.

\section{Méthode}

2.1. Participants et situation 


\section{eJRIEPS 30 juillet 2013}

Cette étude a été conduite en Lycée dans une classe de Seconde durant un cycle de musculation qui comptait sept leçons. Nous nous sommes centrés sur les trois premières dans la mesure où l'enseignant y avait pour objectif de développer des habitudes de travail chez les élèves dans le contexte de la leçon. Les leçons se déroulaient dans deux salles contiguës : dans l'une les élèves devaient réaliser des exercices sur des appareils de musculation différents ; dans l'autre les élèves réalisaient des exercices sans appareil. L'activité de trois élèves volontaires a été étudiée dans la salle appareillée dans laquelle étaient disposés quatre ateliers : le développé couché, la presse oblique, le développé assis et l'appareil à lombaires. Dans la salle se trouvait également un support de charges. Dans chaque atelier, les élèves étaient groupés par quatre, travaillant au sein de deux dyades affinitaires. Dans chaque dyade, les élèves alternaient deux rôles différents : celui « d'athlète » (i.e., l'élève réalisant l'exercice sur l'appareil de musculation), et celui de "pareur » (i.e., l'élève invité à aider et corriger les réalisations de l'athlète). Les élèves avaient dix minutes pour réaliser le travail prescrit par l'enseignant qui annonçait lui-même le changement d'atelier selon un sens de rotation préalablement défini. Dans chaque atelier, le travail consistait à soulever des charges représentant environ $70 \%$ de leur charge maximale, qui avait été évaluée pour chaque élève au cours de la première leçon. Les élèves devaient réaliser trois séries de huit à onze répétitions, avec une récupération de trois minutes entre chaque série. Une fois les trois séries réalisées, un temps d'étirement dans l'atelier avait été préconisé par l'enseignant pour attendre le signal du changement d'atelier. Nous nous sommes intéressés à l'activité des trois élèves lorsqu'ils étaient dans le rôle d'athlète dans la mesure où ils interagissaient avec les appareils de musculation. Ces élèves étaient membres de trois groupes de travail distincts. Nous les avons identifiés respectivement selon les pseudonymes de Chloé, Thomas et Carole.

\subsection{Recueil des données}

Deux catégories de données ont été recueillies : (a) des données d'enregistrement audio et vidéo en classe au cours des trois premières leçons ; (b) des données de verbalisations lors d'entretiens post leçon, à l'issue de la troisième leçon.

Les données d'enregistrement vidéo en classe ont été recueillies grâce une caméra disposée sur un pied, en plan large, permettant de filmer en continu l'ensemble des quatre ateliers et les élèves en activité sur ces ateliers, dont en particulier les comportements de Chloé, Thomas et Carole, au sein de leurs groupes respectifs. Les données d'enregistrement audio en classe ont été obtenues par l'intermédiaire de deux microphones répartis entre les ateliers (le premier entre l'atelier des lombaires et du 


\section{eJRIEPS 30 juillet 2013}

développé couché ; le second entre l'atelier de la presse oblique et du développé assis) permettant d'avoir accès aux échanges verbaux entre les élèves et aux différents bruits potentiellement significatifs pour les élèves présents dans la salle (e.g., bruit des plaques de fonte). Au-delà du recueil de données d'enregistrement audio et vidéo, les deux premières leçons ont permis au chercheur de nouer une relation de confiance avec les élèves, favorable à la conduite des entretiens lors de la troisième leçon.

Les données de verbalisation des trois élèves volontaires ont été recueillies au cours d'entretiens d'autoconfrontation individuels conduits simultanément par trois chercheurs et organisés immédiatement après la troisième leçon. Cette procédure visait à recueillir des données permettant de documenter le cours d'expérience des élèves au cours des trois premières leçons (Theureau, 2004, 2006). Elle a consisté à confronter individuellement chaque élève à l'enregistrement de son activité au sein de son atelier de travail dans chacune des trois leçons, et à l'inviter à montrer, commenter et raconter pas à pas et le plus précisément possible, particulièrement ses actions, ses perceptions, préoccupations et émotions. Ces entretiens, d'une durée moyenne de trente minutes par leçon, se sont déroulés sur le lieu même de la leçon, dans trois salles distinctes.

\subsection{Traitement des données}

Le traitement des données a été réalisé en quatre étapes : (a) la reconstruction des cours d'expérience de chacun des trois élèves, (b) le repérage dans les cours d'expérience d'épisodes d'activité dirigés vers d'autres ateliers, (c) l'identification chez les élèves de préoccupations typiques, lorsqu'ils étaient engagés dans une activité dirigée vers d'autres ateliers et (d) l'identification des actions typiques associées à ces préoccupations typiques.

\subsubsection{Reconstruction des cours d'expérience}

La reconstruction des cours d'expérience a consisté pour chaque élève à identifier les unités significatives pour eux et émergeant de leur interaction avec leur environnement, ainsi que les composantes de chacune d'elles. Les trois composantes qui ont été considérées dans le cadre de cette étude sont l'unité significative élémentaire, le représentamen, et l'engagement dans la situation (Tableau I). La documentation de ces composantes a été conduite grâce à une analyse simultanée des enregistrements audio et vidéo des leçons, des retranscriptions verbatim des verbalisations issues des entretiens d'autoconfrontation, et d'un questionnement spécifique pour chacune d'elles.

L'unité significative élémentaire $(U)$ est la fraction de l'activité qui est montrée, racontée et commentée par l'acteur. Elle peut être une interprétation, un sentiment, une action ou une 


\section{eJRIEPS 30 juillet 2013}

communication (e.g., "Se tourne et regarde en direction de l'atelier «presse »). Elle a été identifiée par un questionnement du type : Que fait l'élève ? Que pense-t-il ? Que ressentt-il ? (de son propre point de vue).

Le représentamen $(\mathrm{R})$ est ce qui, à l'instant $\mathrm{t}$, fait effectivement signe pour l'acteur. II peutêtre un jugement perceptif, mnémonique ou proprioceptif (e.g., "Le bruit des plaques de fonte »). II a été identifié par un questionnement du type : Que prend en compte l'élève ? Quel est l'élément perçu, rappelé ou interprété, qui est significatif pour lui ?

L'engagement $(E)$ dans la situation est la résultante du faisceau d'intérêts de l'acteur à l'instant $t$ et traduit une ouverture/clôture des possibles pour l'acteur au regard de son histoire. L'engagement exprime les préoccupations saillantes en fonction de ce qui fait signe pour l'acteur à un instant précis (e.g., "Rechercher l'origine du bruit »). II a été identifié par un questionnement du type: Quelles sont les préoccupations de l'élève en fonction des éléments pris en compte dans la situation?

2.3.2. Identification des épisodes d'activité dirigés vers des ateliers distants Les épisodes d'activité dirigés vers d'autres ateliers ont été repérés à partir (a) des enregistrements audio et vidéo en situation, (b) du contenu des verbalisations de l'autoconfrontation où l'élève décrivait ou commentait sa prise en compte de l'activité des élèves pratiquant sur les autres ateliers, ou d'événements survenant sur ces ateliers, et (c) de la sélection des unités significatives du cours d'expérience de chaque élève dont les composantes $(U, R, E)$ traduisaient la prise en compte d'éléments d'ateliers distants (e.g., actions d'élèves, bruits). Le Tableau 1 présente un extrait du cours d'expérience de Chloé qui au cours des trois leçons, manifestait régulièrement sa curiosité à s'intéresser à l'activité d'élèves sur d'autres ateliers en regardant autour d'elle. 


\section{eJRIEPS 30 juillet 2013}

Tableau I. Extrait du cours d'expérience de Chloé dirigé vers un atelier distant.

\begin{tabular}{|c|c|c|c|}
\hline & $\begin{array}{l}\text { d'autoconfrontation: "Là, le } \\
\text { le les autres [montre du doigt } \\
\text { en ils mettaient de poids pou } \\
\text { oup parce qu'il avait mis pres } \\
\text { oup de poids avec ses jambe }\end{array}$ & $\begin{array}{l}\text { bruit, c'est le bruit des } \\
\text { sur l'écran l'atelier pre } \\
\text { s'échauffer. Je me dis } \\
\text { ye tout [toutes les ch } \\
\text { et moi non. " }\end{array}$ & $\begin{array}{l}\text { ques à la presse. Je } \\
\text { Je regardais } \\
\text { Arthur il porte } \\
\text { s]. II peut porter }\end{array}$ \\
\hline & $\begin{array}{l}\text { Unités élémentaires } \\
\qquad(U)\end{array}$ & $\begin{array}{c}\text { Représentamen } \\
(\mathrm{R})\end{array}$ & $\begin{array}{c}\text { Engagement } \\
(\mathrm{E})\end{array}$ \\
\hline 60 & $\begin{array}{l}\text { Se retourne subitement vers } \\
\text { l'atelier presse }\end{array}$ & $\begin{array}{l}\text { Le bruit des plaques } \\
\text { dans l'atelier presse }\end{array}$ & $\begin{array}{l}\text { Regarder ce qui se } \\
\text { passe à la presse }\end{array}$ \\
\hline 61 & $\begin{array}{l}\text { Se dit qu'Arthur porte } \\
\text { beaucoup de poids à la } \\
\text { presse }\end{array}$ & $\begin{array}{l}\text { Presque toutes les } \\
\text { plaques sont sur la } \\
\text { presse }\end{array}$ & $\begin{array}{l}\text { Estimer le poids mis } \\
\text { par les élèves pour } \\
\text { s'échauffer }\end{array}$ \\
\hline 62 & $\begin{array}{l}\text { Se dit qu'elle ne pourra pas } \\
\text { soulever la même charge } \\
\text { dans l'atelier presse }\end{array}$ & $\begin{array}{l}\text { La charge soulevée } \\
\text { par Arthur }\end{array}$ & Se comparer à Arthur \\
\hline
\end{tabular}

\subsubsection{Identification des préoccupations typiques}

Une comparaison systématique des préoccupations des élèves dans chacun des épisodes d'activités dirigés vers des ateliers distants a été réalisée. L'ensemble de ces préoccupations a été progressivement réparti au sein de catégories de rangs plus élevées, selon une démarche de comparaison constante inspirée des principes de la Grounded Theory (Strauss \& Corbin, 1990). Ainsi, les 34 préoccupations singulières dénombrées dans les cours d'expérience des trois élèves ont été catégorisées au sein de quatre catégories de préoccupations typiques. Chaque préoccupation typique regroupe un ensemble de préoccupations singulières selon des critères de ressemblance ou de similitude de contenu (Tableau II). 


\section{eJRIEPS 30 juillet 2013}

Tableau II. Exemple de regroupement de préoccupations singulières dans une catégorie de préoccupation typique.

\begin{tabular}{|l|l|}
\hline \multicolumn{1}{|c|}{ Préoccupations singulières } & \multirow{2}{*}{ Préoccupation typique } \\
\hline Estimer la difficulté de l'exercice & \multirow{2}{*}{ Anticiper le travail et les performances } \\
\cline { 1 - 1 } Se comparer aux autres & \\
\cline { 1 - 1 } Préparer la charge à soulever & \\
\cline { 1 - 1 } Contimer la charge soulevée par W. le travail réalisé par W. & \\
\cline { 1 - 1 } Estimer la difficulté de l'exercice & \\
\hline Compter la quantité de répétitions de $\mathrm{K}$. & \\
\hline
\end{tabular}

2.3.4. Identification des actions typiques associées aux préoccupations typiques Chaque préoccupation typique était associée à une action typique. Ces actions typiques, traduisaient, en référence aux catégories génériques définies par Theureau (2006), une catégorie particulière d'unité significative élémentaire, comme par exemple une action pratique, une communication ou une focalisation. Elles ont été identifiées à partir (a) des enregistrements audio et vidéo (e.g., lorsqu'un élève s'adressait verbalement à un autre pour lui demander ce qu'il fallait faire à son futur atelier, qui était une occurrence de la préoccupation typique "anticiper le travail et la performance", l'action typique correspondante était qualifiée de "communication»), et (b) des verbalisations des élèves lorsqu'ils décrivaient eux-mêmes ce qu'ils faisaient lorsqu'ils réagissaient à un évènement significatif pour eux sur un atelier distant (e.g., lorsque Chloé décrivait : " Je regardais si elle y arrivait ou pas. En fait je me compare", cette description traduisait une action typique que nous avons qualifiée de "focalisation", associée à une occurrence de la préoccupation typique « anticiper le travail et la performance »).

\section{Résultats}

Nos résultats mettent en évidence chez les élèves occupant le rôle d'athlète (a) l'existence de quatre préoccupations typiques dirigées vers des ateliers distants, dont les occurrences étaient liées à trois actions typiques dans l'activité des élèves, et (b) des relations entre ces actions typiques et le type d'appareil de musculation présent dans l'atelier.

3.1. Les préoccupations typiques des élèves dirigées vers les ateliers distants et leurs actions typiques 


\section{eJRIEPS 30 juillet 2013}

Quatre préoccupations typiques des élèves dirigées vers les ateliers distants ressortent de l'analyse : (a) « anticiper le travail et les performances », (b) «s'informer », (c) « se mettre en avant », et (d) "s'entraider». Ces préoccupations étaient associées à trois actions typiques dans l'activité des élèves : des focalisations perceptives, des actions pratiques et des communications. Les focalisations perceptives caractérisaient les moments au cours desquels les élèves étaient sensibles à des bruits provenant des ateliers distants, ou lorsqu'ils observaient délibérément les élèves d'un autre atelier de travail. Les actions pratiques caractérisaient des déplacements, des postures ou gestes souvent exécutés de façon ostentatoire. Les communications concernaient les échanges verbaux avec des élèves d'autres ateliers.

\subsection{1. "Anticiper le travail et les performances "}

Les élèves étaient attentifs aux commentaires provenant de camarades sur d'autres ateliers, à propos du travail à réaliser : "Il [Thomas] nous disait que c'était dur [le développé couché] " (Chloé dans l'atelier presse oblique et au sujet de l'atelier développé couché). Ces commentaires étaient pris au sérieux par les élèves qui anticipaient le travail futur à réaliser : "Lui [Thomas] il avait fait l'atelier [développé couché], il sait donc de quoi il parle " (Chloé dans l'atelier presse oblique au sujet de l'atelier développé couché). A travers les commentaires relatifs à la réalisation des tâches sur les ateliers voisins, les élèves anticipaient également le déroulement de la leçon: "J'entends qu'ils se disent qu'ils vont bientôt tourner d'ateliers. Ca va tourner " (Carole à la presse oblique). C'est aussi en balayant du regard les autres ateliers qu'ils étaient amenés à porter une attention particulière aux charges manipulées par les autres élèves et à leurs difficultés pour les soulever. "Je regardais Arthur soulever ses poids (dans l'atelier presse oblique); je savais qu'il n'allait pas y arriver" (Thomas dans l'atelier développé couché). Ils cherchaient ainsi à se renseigner sur les performances réalisées par les autres élèves sur les ateliers voisins : "Je regardais si elles y arrivaient (à propos d'un groupe de filles sur l'atelier développé assis). Si je suis aussi musclé que les filles, enfin, j'y arrive " (Chloé). Les élèves estimaient les performances réalisées par leurs pairs et anticipaient les leurs par comparaison : "Voir un peu les charges qu'ils prennent. Voir si c'est vraiment trop pour éviter de voir trop haut quand je passe après » (Carole).

\subsection{2. "S'informer».}

Les élèves interrogeaient fréquemment à distance un camarade se trouvant sur un autre atelier à propos du travail à réaliser, notamment par des actions pratiques comme mimer un mouvement : "Là je suis en train de lui demander s'il faut bien pousser six fois " 


\section{eJRIEPS 30 juillet 2013}

(Carole depuis l'atelier développé assis vers l'atelier de la presse oblique). Ils questionnaient également verbalement des élèves des ateliers voisins sur le nombre de répétitions : «Elodie! On a combien de répétitions là? (Carole depuis l'atelier développé assis en direction de l'atelier presse oblique), ou sur la façon de mettre en place des charges sur les appareils et la quantité de charges à disposer : «Tu les mets comment [les charges] et t'en mets combien ? (Thomas depuis l'atelier développé couché questionnant Arthur dans l'atelier presse oblique). Les élèves cherchaient également à s'informer sur les évènements inattendus survenant dans la classe. Ils étaient sensibles par exemple aux bruits « anormaux » des plaques de fonte : "Là, on a entendu un gros claquage de poids " (Thomas dans l'atelier lombaires en se tournant vers l'atelier presse oblique). Ce type de bruit évoquait chez eux un incident, ou un problème particulier sur un atelier proche. Dans ce cas ils se détournaient momentanément de leur travail pour s'intéresser à l'atelier d'où provenait le bruit: "Les bruits comme ça, c'est qu'il y a un problème. Alors on regarde ce qui se passe » (Thomas depuis l'atelier lombaires au sujet de l'atelier presse oblique).

\subsection{3. «Se mettre en avant».}

Les élèves cherchaient à certains moments à se valoriser ou à manifester ostensiblement la supériorité de leurs performances en comparaison de celles des autres, en particulier lors de communications verbales avec les autres : "Là je suis en train de lui dire: C'est tout ? [Ce que tu soulèves]. II sait que je porte beaucoup de poids avec mes jambes et lui non!» (Thomas depuis l'atelier développé couché en interpellant ironiquement un élève de l'atelier presse oblique) ; ou par des actions pratiques comme attirer le regard d'autres camarades afin de démontrer publiquement leurs performances physiques : «Là, je faisais un peu le guignol pour amuser la galerie et que les filles me regardent. Qu'elles voient ce que je soulève quoi! » (Thomas).

3.1.4. « S'entraider».

Les élèves aidaient parfois un élève d'un autre atelier à réaliser le travail à faire à travers des communications : "Je lui ai indiqué quels poids il fallait prendre " (Thomas, depuis l'atelier lombaires, s'adressant à un élève travaillant dans l'atelier de développé assis). C'est aussi en relation avec leur perception des réactions des autres que des élèves trouvaient des sources de motivation nécessaires à la réalisation ou la poursuite du travail à réaliser. Ils percevaient en particulier les encouragements d'élèves d'ateliers voisins, qui stimulaient leur investissement: "Là il me dit que c'est balaise [de porter une charge aussi importante]. Je me dis que c'est un compliment " (Thomas dans l'atelier développé 


\section{eJRIEPS 30 juillet 2013}

couché recevant les encouragements d'un élève de l'atelier presse oblique). Cet investissement était parfois lié à leur sensibilité au «bruit de fond»constitué par les conversations entre élèves dans la salle. "Parfois, j'écoute ce que les autres se racontent ; ça fait comme un bruit de fond » (Thomas). Ce bruit de fond qui accompagnait le travail des élèves était considéré comme une aide par eux : " Je me sens plus à l'aise lorsque je travaille et que j'entends les autres à côté » (Thomas). A l'inverse, lorsqu'ils ne le percevaient plus, ils se sentaient alors moins à l'aise : "Tout seul ça fait bizarre. C'est mieux quand il y a du monde autour »(Chloé).

3.2. Les relations entre les actions typiques associées aux préoccupations typiques et l'appareil présent dans l'atelier

Les élèves ne manifestaient pas leurs préoccupations vis-à-vis des autres ateliers selon les mêmes actions typiques en fonction du type d'appareil présent dans l'atelier: l'expression de ces actions typiques était indexée aux caractéristiques physiques des appareils.

Dans les ateliers presse oblique et développé couché, les élèves devaient s'allonger sur le dos, le regard orienté vers le haut pour réaliser les exercices. Cette posture corporelle ne leur permettait pas de regarder aisément autour d'eux : " Je ne me rends pas compte sur cette activité là (la presse oblique) parce que je vois mes pieds. En fait, je ne vois pas » (Chloé). Elle limitait leurs possibilités de communications verbales et de déplacements vers d'autres ateliers (ceux-ci n'étaient possibles qu'après avoir réalisé les exercices). Une fois engagés dans leur travail, les élèves préféraient rester en position allongée pendant les temps de récupération entre les séries. Ils ne se relevaient qu'après avoir réalisé la totalité de l'exercice, et pouvaient alors se déplacer vers un atelier voisin soit pour communiquer avec d'autres élèves, soit pour répondre à la demande de l'enseignant qui ordonnait une rotation entre les ateliers. Par contre cette posture allongée les invitait à être plus attentifs à la réalisation de l'exercice : «Là je regarde la barre. Je regarde les poids » (Thomas au développé couché) ; davantage « repliés sur eux-mêmes » : « Là je ne voyais pas. J'étais dans mon truc" (Thomas au développé couché). Cette sensation de « repli sur soi » donnait parfois l'impression aux élèves d'être particulièrement observés : "Je ne les [les filles dans l'atelier presse oblique] vois pas mais je sens qu'elles parlent de moi, qu'elles m'observent. Je sens des regards sur moi » (Thomas en développé couché). Toutefois ils restaient sensibles aux échanges verbaux dans les ateliers proches : «J'entends les filles papoter [dans l'atelier presse oblique] » (Thomas en développé couché). Lorsque les élèves réalisaient les exercices sur les appareils de la presse oblique 


\section{eJRIEPS 30 juillet 2013}

et du développé couché, ils manifestaient leurs préoccupations principalement par des focalisations perceptives sur les échanges verbaux ou les observations d'élèves sur d'autres ateliers. Dans les ateliers lombaires et développé assis, les élèves étaient soit dans une posture assise (en développé assis), soit semi-allongée avec la tête relevée vers l'avant et le haut (appareil à lombaires). Ces deux postures corporelles leur offraient des conditions favorables pour prêter attention aux évènements sur l'ensemble des ateliers. Tout en réalisant les exercices, ils en profitaient pour observer les élèves travaillant sur les autres ateliers: "Ici (atelier lombaire) on voit bien ce qui se passe. On peut même voir les autres à la presse. J'aime bien regarder autour de moi " (Chloé). Le fait d'être en position assise ou semi-allongée au cours des séries (contrairement aux ateliers de presse oblique et de développé couché, dans lesquels les élèves étaient en position allongée) permettait aux élèves de se déplacer plus facilement. Ainsi ils quittaient parfois leur appareillage de musculation pour se rapprocher d'un atelier voisin, même lors des moments de récupération entre les séries: "On se rapprochait par curiosité. Pour entendre ce que disait Thomas à Elodie " (Chloé à la presse oblique). D'autres fois, tout en réalisant un exercice, ils questionnaient un camarade se trouvant sur un atelier distant, ou cherchaient à attirer l'attention des élèves des ateliers voisins. Lorsque les élèves réalisaient les exercices sur les appareils à lombaires et du développé assis, ils manifestaient leurs préoccupations principalement sous la forme d'actions pratiques les amenant parfois à quitter leur propre atelier, et de communications verbales avec des élèves plus ou moins proches sur l'ensemble des autres ateliers.

\section{Discussion}

Nos résultats montrent que, du point de vue de l'expérience des élèves, chaque atelier de travail ne constitue pas un isolat. Une partie de l'activité des élèves est, bien qu'engagée dans la réalisation d'une tâche particulière dans un atelier, conjointement dirigée vers l'activité des élèves dans les autres ateliers de la classe. Cette activité, dont les actions typiques dépendent des dispositifs matériels utilisés, génère diverses sortes d'interactions entre les élèves d'ateliers distants. Ces résultats sont interprétés en deux points. Le premier discute le potentiel de ressources distribuées sur les ateliers. Le deuxième point envisage des pistes pour l'intervention en considérant les interactions des élèves entre les ateliers comme une aide possible pour l'enseignant.

4.1. Les ressources potentielles et distribuées dans un format pédagogique en atelier 


\section{eJRIEPS 30 juillet 2013}

Dans un format en ateliers, l'activité des élèves ne se réduit pas à la réalisation du travail assigné par l'enseignant à leur atelier. Cette activité intègre, pour une large part, des interactions avec des élèves présents sur des ateliers voisins. Ces interactions qui prennent des formes variées (focalisations perceptives, actions pratiques et communications) se caractérisent par leur caractère spontané et par les liens étroits qu'elles entretiennent avec les objets matériels présents dans chaque atelier. Nous suggérons que, du point de vue de l'activité des élèves, les ateliers ne peuvent pas être considérés indépendamment les uns des autres, mais constituent un véritable réseau de ressources pour leurs apprentissages. Bien que répartis à différents endroits dans l'espace de la leçon, nos résultats montrent que dans ce contexte inévitablement collectif et public (Doyle, 1986) les élèves trouvent «dans " et "par » l'activité d'autrui, des opportunités pour développer des expériences vicariantes (Bandura, 1980) (e.g., estimer ses propres possibilités de répondre aux exigences d'un atelier grâce à l'observation des performances des autres) et pour s'aider à réaliser le travail prescrit par l'enseignant dans leur atelier ou encore se préparer au travail à venir. C'est par exemple en interagissant depuis leur atelier par des questionnements ou des gestuels, par l'observation ou l'écoute attentive d'un élève en train de réaliser les exercices sur un atelier distant, que les élèves anticipent le travail et les performances ou s'encouragent pour réussir la tâche. Mais les appareils de musculation ne maintenaient pas toujours les élèves dans les ateliers. Certains quittaient leur espace de travail pour se déplacer sur un autre atelier afin de s'informer ou conseiller un camarade. Les évènements (e.g., la démonstration ostentatoire d'une performance) ou les éléments du contexte matériel (e.g., les appareillages de musculation) de chaque atelier constituent pour les élèves autant de ressources potentielles distribuées sur l'ensemble de l'espace de la leçon. Ces ressources, lorsqu'elles sont exploitées par les élèves, participent à l'émergence d'interactions qui prennent la forme "d'interactions à distance mutuellement bénéfiques " pour les élèves. Ces interactions sont qualifiées de «mutuellement bénéfiques " parce que, d'une part, elles permettent aux élèves de «tirer leur épingle du jeu » dans la situation dans laquelle ils sont engagés, et d'autre part, elles favorisent l'émergence de diverses formes de tutelle ou de travail coopératif spontanés entre les élèves (Huet \& Saury, 2011). Lorsqu'il s'agit par exemple de s'informer sur le travail à réaliser, de se conseiller, de trouver des explications pour comprendre les modalités d'utilisation des autres ateliers, chaque élève adopte spontanément un rôle particulier (e.g., conseilleur vs conseillé), et vit une expérience d'apprentissage coopératif (e.g., apprendre à donner des conseils adaptés aux 


\section{eJRIEPS 30 juillet 2013}

problèmes rencontrés par son partenaire ; ou accepter, faire confiance et prendre à son compte les conseils d'un pair pour réussir la tâche). Toutefois ces interactions n'ont pas systématiquement une dimension coopérative : certaines sont par exemple l'occasion de démonstrations ostentatoires de certains élèves visant à attirer l'attention des autres sur leurs performances, accentuer la comparaison sociale, ou stimuler des moqueries. Dans ces moments, l'éloignement de l'enseignant peut être perçu par les élèves comme une opportunité pour actualiser ces interactions. Dans le cas de notre situation d'étude, en raison du fait que l'espace de la leçon intégrait deux salles contigües, l'enseignant ne pouvait pas avoir un contrôle sur tous les élèves de la classe. La distance le séparant des élèves est un donc un des éléments ayant participé à définir la nature des interactions entre les élèves. Si des travaux récents ont pointé les liens entre le caractère masqué ou ostentatoire de l'activité des élèves et la distance les séparant de l'enseignant (Vors \& Gal-Petitfaux, 2011), nous devons considérer l'apparition d'interactions à distance mutuellement bénéfiques comme étant aussi indexée à la proximité/éloignement de l'enseignant des élèves. Cependant, nos résultats accréditent l'idée selon laquelle la classe forme un système éco-social, au sein duquel les agencements matériels, symboliques et sociaux constituent des « réseaux d'opportunités/possibilités d'actions 》 (« affordance networks ») (Barab \& Roth, 2006). Lors des leçons, les élèves semblent tirer bénéfice de tous les possibles de l'environnement de la leçon pour s'entraider. Mais ces «affordance networks» ne sont pas des propriétés objectives de l'espace et de la matérialité physique de la leçon. Ils sont au contraire indissociables des objets matériels présents dans chaque atelier et qui participent à structurer l'activité des élèves. A titre d'illustration, la posture corporelle des élèves lorsqu'ils sont au « développé couché » les amène à être plus sensibles aux échanges verbaux des ateliers voisins, qui constituent pour eux un «bruit de fond » stimulant la réalisation des exercices. Dans le même temps, ces ateliers particuliers limitent leurs possibilités de communiquer avec les élèves des ateliers distants, ou d'interagir gestuellement avec eux. Les ateliers sont donc «connectés » entre eux par des interactions à distance potentiellement mutuellement bénéfiques. Celles-ci émergent conjointement des offres de l'environnement et de l'activité des élèves, médiées par la matérialité de chaque atelier. Les appareils de musculation, et plus particulièrement leurs caractéristiques physiques, jouent le rôle de médiateurs dans l'émergence de ces interactions. Ainsi, c'est à travers ces médiations matérielles (Adé \& de Saint Georges, 2010; Jonassen \& Land, 2000; Norman, 1993; Vinck, 1999; Winn, 2002) que se structurent entre les ateliers des opportunités d'échanges, d'aides, 


\section{eJRIEPS 30 juillet 2013}

d'encouragements mais aussi de mise en valeur des performances. En pointant ces interactions à distance mutuellement bénéfiques, nos résultats viennent confirmer ceux d'études antérieures ayant mises en avant l'existence d'interactions coopératives spontanées au sein de dyades symétriques et dissymétriques (Darnis, Lafont, \& Menaut, 2005; Gernigon, Huet, Winnykamen, \& Cadopi, 2002). Ils sont également convergents avec ceux de l'étude de Huet \& Saury (2011), qui ont montré que les configurations d'activité collective générées par un dispositif de travail par équipe en athlétisme offraient aux élèves un ensemble de ressources distribuées pour apprendre, et engendrait des formes originales de coopération et de tutelle non prescrites par l'enseignant. Cependant, il s'agissait dans cette étude d'interactions entre élèves d'un même groupe, sur un même atelier athlétique. Nos résultats mettent en évidence que des phénomènes comparables peuvent aussi apparaître entre des ateliers distants, et pas seulement au sein d'un même atelier.

\subsection{Pistes pour l'intervention}

En pointant les liens entre l'environnement spatial et matériel de la leçon et les interactions à distance entre élèves, nos résultats nous invitent à réfléchir à l'idée d'une ergonomie de la conception des dispositifs d'apprentissage en EPS centrée sur les cours d'expérience des élèves (Saury et al., 2013). Cette ergonomie chercherait à comprendre l'activité des élèves afin de concevoir ou d'optimiser des apprentissages pertinents du point de vue de leurs visées pédagogiques. Pour concrétiser cette idée dans le cadre de notre étude, nous proposons par exemple aux enseignants, dans des formats pédagogiques en ateliers, d'anticiper les interactions des élèves en lien avec la matérialité de chaque atelier et l'arrangement spatial des ateliers, et de prendre au sérieux ce qui se « joue » entre les ateliers. Concevoir une organisation de travail en ateliers, c'est proposer aux élèves un "réseau d'interactions encouragées ". Cette idée est consistante avec celle d'" espace d'actions encouragées » (Bril, 2002; Durand, 2008) car respectant le potentiel de toutes formes d'activité émergentes. La notion d'espace d'actions encouragées (Reed \& Bril, 1996) a pour but "d'introduire dans l'espace d'action un ensemble d'activités, d'objets, de lieux qui doivent amener l'apprenant à augmenter son potentiel d'actions, et par voie de conséquences, ses capacités d'actions " (Bril, 2002, p.258). Mais l'idée de "réseau d'interactions encouragées ", au-delà des dimensions spatiales, temporelles et matérielles, intègre les interactions (entre élèves et entre les élèves et l'enseignant). Un "réseau d'interactions encouragées » caractérise un dispositif d'apprentissage englobant sans les disjoindre les formes de groupement des élèves, les agencements des ateliers 


\section{eJRIEPS 30 juillet 2013}

dans l'espace, leurs composants matériels et les modalités d'intervention de l'enseignant, le tout étant susceptible de stimuler des interactions mutuellement bénéfiques. Autrement dit, dans un format en ateliers, il est possible sous certaines conditions, d'encourager ou de limiter certaines interactions chez les élèves. Au niveau des formes de groupement, une voie possible est de composer au sein de la classe des dyades d'élèves (athlète et pareur) engagées dans des attentes communes relativement à un projet, mais ne partageant pas le même atelier de travail. Cette forme de groupement des élèves, dans laquelle ces derniers seraient éloignés spatialement et s'exerceraient sur des appareils de musculation différents, tout en partageant un horizon d'attentes commun, pourrait favoriser l'attention mutuelle entre les élèves au sein des dyades. Au niveau des dimensions spatiales et matérielles nous proposons, pour concevoir des leçons de musculation dans un format en ateliers, de prendre en compte simultanément les offres des appareillages et leur agencement spatial. Prendre en compte les offres des appareillages c'est passer d'une classification centrée sur les groupes musculaires ou les actions motrices à réaliser, à une classification centrée "activité ". Nous pouvons alors distinguer deux types d'appareillages : ceux limitant les interactions à distance (e.g., le développé couché) et ceux les stimulant (e.g., développé assis). Dans le but d'agencer les appareillages de musculation en fonction du potentiel d'interactions à distance qu'ils offrent, nous proposons par exemple de les aligner en intercalant ceux qui stimulent les formes d'interactions à distance avec ceux qui les limitent. Nous pensons ainsi réduire les possibles ouverts par une organisation en face à face qui invite les élèves sur les appareillages stimulant les interactions à distance à prendre en compte les évènements dans tout l'espace de la leçon. Cette organisation spatiale et matérielle de l'espace de la leçon peut être considérée comme complémentaire à la préoccupation de stimuler des interactions coopératives entre les élèves au sein de chaque atelier.

II s'agit donc d'encourager les interactions à valence positive car utiles au bon déroulement de la leçon et aux apprentissages des élèves, et de dissuader celles à valence négative car source de perturbations. Pour cela nous posons trois principes en lien avec notre inscription dans le cadre théorique du cours d'action (Theureau, 2004, 2006) : le principe du pari, de la non-prescription et le principe d'aide. Le principe du pari signifie que du fait du caractère autonome de l'activité, un réseau d'interactions encouragées ne peut offrir les garanties que les significations construites par l'enseignant à travers l'arrangement spatial ou le choix des objets matériels soient effectivement partagées avec les élèves. Le principe de la non-prescription signifie qu'on ne peut obliger 


\section{eJRIEPS 30 juillet 2013}

les élèves à s'aider, s'encourager ou s'informer à distance. C'est l'acteur qui définit ce qui pour lui est significatif dans un environnement. Si le but de l'enseignant est de maintenir en activité les élèves dans chaque atelier, il ne peut que chercher à encourager les interactions à distance à valence positive. Enfin le principe d'aide signifie que le réseau d'interactions encouragées doit participer au bon déroulement de la leçon. L'aide est donc aussi bien pour les élèves (e.g., s'informer sur le travail à réaliser, s'encourager) que pour l'enseignant (e.g., éviter la répétition des consignes, se délester de certaines régulations, limiter les déplacements entre les ateliers). II ne s'agit en aucun cas de prétendre garantir des interactions à valence positive dans la mesure où leur qualité est liée à leur caractère spontané, émergent. L'enseignant doit être envisagé comme un « chef d'orchestre » qui met en forme l'activité collective des élèves dans le contexte de la leçon, mais dont le résultat découle de la dynamique des couplages entre les acteurs de la leçon (enseignant et élèves) et les éléments qui composent cet environnement (les objets de leçon et leur arrangement spatial).

\section{Conclusion}

Bien qu'il faille relativiser la portée de nos résultats en raison du faible nombre d'élèves qui limite leurs possibilités de généralisation, nous pensons que cette étude contribue à enrichir les connaissances relatives aux interactions spontanées entre les élèves, et à l'inscription contextuelle des situations d'enseignement et d'apprentissage. Concevoir les formats pédagogiques en ateliers en termes de réseaux d'interactions médiés par les objets matériels de la leçon nous invite également à penser les leçons d'EPS en termes de «partage d'informations contextuelles » (Salembier \& Zouinar, 2004, 2006) ou de « contexte mutuellement partagé » (Dumazeau, 2005). Bien que ces notions soient issues de travaux en ergonomie et sans lien direct avec l'enseignement, elles nous semblent être des sources d'inspirations fécondes pour la compréhension des situations d'enseignement / apprentissage dans la mesure où ces approches conçoivent les processus de partage mutuel dans une conception non déterministe, découlant de la co-construction entre les interactions des acteurs et l'environnement dans lequel elles se déploient.

\section{Bibliographie}

Adé, D., \& de Saint Georges, I. (2010). Les objets dans la formation. Usages, rôles et significations dans des situations variées. Toulouse: Octarès. 


\section{eJRIEPS 30 juillet 2013}

Adé, D., Jourand, C., \& Sève, C. (2010). L'inscription contextuelle de l'activité en course en durée : une étude à partir de l'analyse de l'activité d'élèves de primaire en Education Physique et Sportive. Education \& Didactique, 4(3), 7-19.

Adé, D., Veyrunes, P., \& Poizat, G. (2009). Les objets dans l'activité interindividuelle en classe : l'exemple de leçons d'Education Physique et Sportive et de géographie. Travail et Apprentissage, 3(1), 124-139.

Gernichon C., Huet, M.L., Cadopi, M., \& Winnykamen, F. (2002). Peer tutoring in a physical education setting: Influence of tutor skill level on novice learners' motivation and performance. Journal of Teaching in Physical Education, 22(1), 105-123.

Bandura, A. (1980). L'apprentissage social. Bruxelles: éditions Pierre Mardaga.

Barab, S. A., \& Roth, W.M. (2006). Curriculum-based ecosystems: Supporting knowing from an ecological perspective. Educational Researcher, 35(5), 3-13.

Bril, B. (2002). Apprentissage et contexte. Intellectica, 2(35), 251-268.

Cizeron, M., \& Gal-Petitfaux, N. (2006). “ Le travail en "vagues” et en "ateliers": deux façons d'enseigner et d'apprendre au cours de leçons de gymnastique ». In $G$. Carlier, D. Bouthier, \& G. Bui-Xuan (Éd.), Intervenir en éducation physique et en sport, (pp. 344-351). Louvain-la-Neuve, Belgique: Presses universitaires de Louvain.

Darnis, F., Lafont, L., \& Menaut, A. (2005). Interactions dyadiques et niveau opératoire pour la construction de stratégies en handball chez des participants de 11-12 ans. Revue Européenne de Psychologie Appliquée/European Review of Applied Psychology, 55(4), 255-265.

De Keukelaere, C., Guérin, J, \& Saury, J. (2008). Co-construction de connaissances chez les élèves en EPS au cours d'une situation d'apprentissage en volley-ball. STAPS, 79(1), 23-38.

Doyle, W. (1986). Classroom organization and management. In Handbook of research on teaching. ed. M.C. Wittrock, 392-431. New York, NJ: Macmillan.

Dumazeau, C. (2005). Rôle du contexte mutuellement partagé dans les communications professionnelles distantes. Langage et société, 125, 55-75.

Durand, M. (1998). Physical education teachers'action analysis: a cognitive anthropology approach. The AIESEP Newsletter, 61, 2-10.

Durand, M. (2001). Chronomètre et survêtement. Reflets de l'expérience quotidienne d'enseignants en éducation physique. Paris: Éditions Revue EPS. 


\section{eJRIEPS 30 juillet 2013}

Durand, M. (2008). Un programme de recherche technologique en formation des adultes. Une approche enactive de l'activité humaine et l'accompagnement de son apprentissage /développement. Education \& Didactique, 3(2), 69-93.

Dyson, B., Griffin, L.L., \& Hastie, P. (2004). Sport Education, Tactical Games, and Cooperative Learning: Theoretical and pedagogical considerations. Quest, 56(2), 226-240.

Dyson, B., \& Grineski, S. (2001). Using cooperative learning structures to achieve quality physical education. Journal of Physical Education, Recreation \& Dance, 72(2), 2831.

Ensergueix, P.J., \& Lafont, L. (2010). Reciprocal peer tutoring in a physical education setting: influence of peer tutor training and gender on motor performance and selfefficacy outcomes. European Journal of Psychology of Education, 25(2), 222-242.

Ensergueix, P.J., \& Lafont, L. (2011). Impact of Trained versus Spontaneous Reciprocal Peer Tutoring on Adolescent Students. Journal of Applied Sport Psychologie, 23(4), 381-397.

Evertson CM, \& Weinstein CS (2006) Classroom management as a field of inquiry. In Evertson CM, \& Weinstein CS (Éd.), Handbook of classroom management: Research, practice, and contemporary issues (pp.3-16). Mahwah, NJ: Lawrence Erlbaum Associates,.

Gal-Petitfaux, N., \& Vors, O. (2010). Le rôle des objets dans l'articulation d'activités publiques et masquées participant à la viabilité d'une situation d'enseignement : une étude en gymnastique scolaire. In D. Adé, \& I. de Saint Georges (Éd.), Les objets dans la formation. Usages, rôles et significations dans des situations variées (pp.161-187). Toulouse: Octarès.

Gal-Petitfaux, N., \& Durand, M. (2001). L'enseignement de l'éducation physique comme «action située»: propositions pour une approche d'anthropologie cognitive. STAPS, 22(55), 79-100.

Gal-Petitfaux, N., Sève, C., M. Cizeron, \& Adé, D. (2010). Activité et expérience des acteurs en situation: les apports de l'anthropologie cognitive. In M Musard, M Loquet, \& G Carlier (Éd.), Sciences de l'intervention en EPS et en sport: résultats de recherches et fondements théoriques (pp. 67-87). Paris: Éditions Revue EPS.

Guillou, J., \& Durny, A. (2008). Students' situated action in physical education: analysis of typical concerns and their relations with mobilized knowledge in table tennis. Physical Education \& Sport Pedagogy, 13(2), 153-169. 


\section{eJRIEPS 30 juillet 2013}

Hastie, P., \& Siedentop, D. (1999). An ecological perspective on physical education. European Physical Education Review, 5(1), 9-29.

Hastie, P., \& Siedentop, D. (2006). The classroom ecology paradigm. In D. Kirk, D. Macdonald, \& M. O'Sullivan (Éd.), The handbook of physical education (pp. 214225). London: Sage.

Huet, B. \& Saury, J. (2011). Ressources distribuées et interactions entre élèves au sein d'un groupe d'apprentissage : une étude de cas en éducation physique et sportive. eJRIEPS, 24, 4-30.

Hutchins, E. (1995). Cognition in the wild. Cambridge, MA: MIT Press.

Jonassen, D.H., \& Land, S.M. (2000). Theoretical foundations of learning environments. Mahwah, NJ: Lawrence Erlbaum Associates.

Kirk, D., and D. Macdonald. 1998. Situated Learning in Physical Education. Journal of Teaching in Physical Education, 17, no. 3: 376-387.

Kirshner, D., \& Whitson, J.A. (1997). Situated cognition: Social, semiotic, and psychological perspectives. Mahwah, NJ: Lawrence Erlbaum Associates.

Lave, J., \& Wenger, E. (1991). Situated learning: Legitimate peripheral participation. Cambridge, MA: Cambridge University Press.

Lave, J. 1988. Cognition in practice. Mind, mathematics and culture in everyday life. Cambridge, MA: Cambridge University Press.

Lincoln, Y. S., \& Guba, E.G. (1985). Naturalistic Inquiry. Beverly Hills, CA: Sage Publications.

Lotan, R.A. (2006). Managing groupwork. In Handbook of Classroom Management: Research, Practice, and Contemporary Issues. ed. Evertson, C. and C. Weinstein, 525-539. Lawrence Erlbaum Associates, Inc. New Jersey.

Maturana, H. R., \& Varela, F.J. (1987). The tree of knowledge: The biological roots of human understanding. Boston: Shambhala Publications.

Norman, D.A. (1993). Things that make us smart. New York: Addison Wesley.

Reed, E., \& Bril, B. (1996). The primacy of action in development. A commentary of N. Bernstein. In M. Latash, \& M. Turvey (Éd.), Dexterity and its development (pp. 431-451). Hillsdale, NJ: Erlbaum Associates.

Robbins, P., \& Aydede, M. (2008). Cambridge handbook of situated cognition. New York: Cambridge University Press. 


\section{eJRIEPS 30 juillet 2013}

Rovegno, I. (2006). Situated perspectives on learning. In D. Kirk, D. Macdonald, \& M. O'Sullivan (Éd.), The handbook of physical education (pp. 262-274). London: Sage.

Salembier, P., \& Zouinar, M. (2004). Intelligibilité mutuelle et contexte partagé. Inspirations conceptuelles et réductions technologiques. @ctivités, 1(2), 64-85.

Salembier, P., \& Zouinar, M (2006). Pas de coopération sans partage! Le partage d'information comme régulateur de la cognition individuelle et collective. In $\mathrm{F}$. Jeffroy, J. Theureau, \& Y. Haradji (Éd.), Relation entre activité individuelle et activité collective: Confrontation des différentes démarches d'études (pp. 55-75). Toulouse: Octarès.

Salomon, G., \& Perkins, D.N. (1998). Individual and social aspects of learning. Review of Research in Education, 23(1), 1-24.

Saury, J., Adé, D., Gal-Petitfaux, N., Huet, B., Sève, C. \& Trohel, J. (2013). Actions, significations et apprentissages en EPS. Une approche centrée sur les cours d'expériences des élèves et des enseignants. Paris : Éditions Revue EPS.

Saury, J., Huet, B., Rossard, C., \& Sève, C. (2010). Dispositifs de co-observation et configurations d'activités en éducation physique et sportive. In . D. Adé, \& I. de Saint Georges (Éd.), Les objets dans la formation. Usages, rôles et significations dans des situations variées (pp.143-159). Toulouse: Octarès.

Saury, J., \& Rossard, C. (2009). Les préoccupations des élèves durant des tâches d'apprentissage coopératives et compétitives en badminton: une étude de cas. Revue des sciences de l'éducation, 35(3), 195-216.

Strauss, A., \& Corbin, J. (1990). Basics of qualitative research. London: Sage.

Suchman, L. (1987). Plans and situated actions: the problem of human machine interaction. Cambridge: Cambridge University Press.

Theureau, J. (2004). Le cours d'action : Méthode élémentaire. Toulouse : Octarès.

Theureau, J. (2006). Le cours d'action: Méthode développée. Toulouse : Octarès.

Varela, F.J. (1989). Autonomie et connaissance. Essai sur le vivant. Paris: Seuil.

Varela, F.J., \& Shear, J., (1999). First-person methodologies: why, when and how. J.Consc.Studies, 6 (2), 1-14.

Vinck, D. (1999). «Les objets intermédiaires dans les réseaux de coopération scientifique. Contribution à la prise en compte des objets dans les dynamiques sociales ». Revue française de sociologie, 40(2), 385-414. 


\section{eJRIEPS 30 juillet 2013}

Vors, O, \& N. Gal-Petitfaux (2011). Situations de travail par ateliers et configuration de l'activité collective en classe « Réseau Ambition Réussite ». eJRIEPS, 22, 96-116.

Ward, P., \& M.A. Lee. (2005). Peer-assisted learning in physical education: A review of theory and research. Journal of Teaching in Physical Education, 24(3), 205-225.

Whitson, J.A. (1997). Cognition as a semiotic process: From situated mediation to critical reflective transcendence. In D. Kirshner, \& J.A. Whitson (Éd.), Situated cognition: Social, semiotic and psychological perspectives (pp. 97-149). Mahwah, NJ: Lawrence Erlbaum.

Winn, W. (2002). Current trends in educational technology research: The study of learning environments. Educational Psychology Review, 15(4), 331-351. 